\title{
Cirurgia reconstrutiva com retalho cutâneo de avanço como técnica alternativa para tratamento de carcinoma de células escamosas em cães: relato de caso*
}

\section{Reconstructive surgery with skin flap advance as alternative technique for treating squamous cell carcinoma in dog: case report}

\author{
Maria Isabel Mello Martins, ${ }^{* *}$ Bruno César Elias, ${ }^{* * *}$ Rebeca Cordeiro Justino, ${ }^{* * *}$ Carmen Lúcia Scortecci Hilst ${ }^{\star * * * *}$
}

\begin{abstract}
Resumo
O carcinoma de células escamosas (CCE) é uma das neoplasias mais frequentemente observadas no trato genital de cães. O tratamento de eleição para o CCE é a exérese com ampla margem cirúrgica $(1 \mathrm{a} 3 \mathrm{~cm})$, porém quando a região prepucial está comprometida, se torna difícil obter margem adequada, tanto pela anatomia da região quanto pelas características agressivas dessa neoplasia. A penectomia com uretrostomia pré-escrotal seria a técnica mais indicada nesses casos, entretanto, complicações pós-cirúrgicas podem reduzir a qualidade e a expectativa de vida do animal. O objetivo deste relato foi demonstrar uma técnica cirúrgica conservativa, com base em plásticas reconstrutivas com retalhos cutâneos, como tratamento para CCE em prepúcio e região inguinal, associada ao uso do piroxicam como terapia adjuvante. O animal não apresentou recidiva do tumor ou complicações secundárias ao tratamento até a última avaliação, um ano após o término do tratamento.
\end{abstract}

Palavras-chave: canino, cirúrgica reconstrutiva, neoplasia, região inguinal, piroxicam.

\begin{abstract}
Squamous cell carcinoma (SCC) is one of the most frequently neoplasms observed in genital tract in dogs. The treatment of choice for the CCE is the excision with wide surgical margin $(1-3 \mathrm{~cm})$, but when the foreskin region is committed becomes difficult to obtain adequate margin, due both the anatomy of the region and the aggressive characteristics of this neoplasm. The penectomy with pre-scrotal urethrostomy would be the most suitable technique in such cases, however, postoperative complications may reduce the quality and expectancy of life. The objective of this report was to present a conservative surgical technique, based on reconstructive plastic with skin flap as a treatment for SCC in prepuce and inguinal region, associated with the use of piroxicam as adjuvant therapy. The dog was evaluated during one year after the end of treatment and haven't showed any tumor recurrence or secondary complications to treatment.
\end{abstract}

Keywords: canine, cosmetic surgical, inguinal region, neoplasia, piroxicam.

\section{Introdução}

Dentre as neoplasias que acometem o trato genital masculino, o carcinoma de células escamosas (CCE) é uma das mais frequentes, correspondendo a $7 \%$ das neoplasias epiteliais em cães e, apesar da maior ocorrência em pacientes geriátricos, há relatos em jovens (Vail e Withrow, 2007). A falta de pigmentação na derme e a frequente exposição solar parecem ser fatores predisponentes (Souza et al., 2006; Vail e Withrow, 2007; Rodaski e Werner, 2008). Inicia-se como uma dermatose solar nas junções mucocutâneas e/ou na pele, com eritema, edema, descamação, formação de úlceras e crostas e áreas mais firmes devido à invasão da derme (Rocha et al., 2010).

O CCE possui comportamento invasivo e infiltrativo; entretanto, existem relatos de metástase em linfonodo regional e pulmão
(Mccaw et al., 2000). A citologia aspirativa com agulha fina (CAAF) auxilia o diagnóstico, mas a confirmação deve ser realizada pelo exame histopatológico (Vail e Withrow, 2007).

As opções terapêuticas incluem cirurgia, criocirurgia, radiação ionizante, terapia fotodinâmica, quimioterapia e, recentemente, os inibidores COX-2 seletivos (Straw, 1998; Holmberg, 2007; Joseph et al., 2008; Alves et al., 2011).

O tratamento de eleição para o CCE é a exérese com ampla margem cirúrgica (1 a $3 \mathrm{~cm}$ ), tendo efeito curativo em muitos casos. Quando a região prepucial está comprometida, torna-se difícil obter margem adequada, sendo necessária a penectomia e uretrostomia pré-escrotal, seguida pela terapia adjuvante (Mestrinho et al.,2012). Complicações pós-operatórias, como hemorragia difusa e deiscência de sutura, e as infecções

*Recebido em 2 de julho de 2014 e aceito em 18 de novembro de 2015.

**Docente do Departamento de Clínicas Veterinárias, CCA - Universidade Estadual de Londrina - UEL, Londrina-PR, Brasil. Autor para correspondência: imartins@uel.br

${ }^{* * *}$ Residente em Medicina Veterinária, área de Teriogenologia de Animais de Companhia, Departamento de Clínicas Veterinárias, CCA - Universidade Estadual de Londrina - UEL, Londrina-PR, Brasil.

****Doutoranda em Ciência Animal, Departamento de Clínicas Veterinárias CCA - Universidade Estadual de Londrina - UEL, Londrina-PR, Brasil.

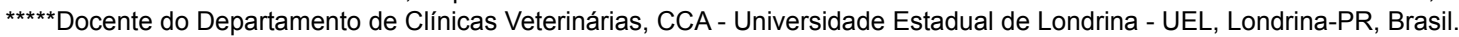


urinárias ascendentes podem ocorrer devido à tensão na pele, decorrente de extensa excisão de tecido acometido (Smeak e Newton, 1998; Smeak, 2000).

As técnicas de cirurgia plástica reconstrutiva podem ser uma opção para evitar as complicações citadas acima e ainda permitir a retirada da neoplasia com ampla margem. Diversos trabalhos citam as vantagens do uso dessas técnicas (Pargana, 2009; Szentimrey, 1998; Sakuma et al., 2003). Fatores como o tipo e grau do tumor, localização anatômica e extensão da invasão local, ditarão a ressecção apropriada e as opções reconstrutivas, como o retalho cutâneo por avanço, transposição e padrão axial, ou através de enxertos cutâneos (Sakuma et al., 2003; Szentimrey, 1998).

Este trabalho teve como objetivo relatar um tratamento conservativo baseado em plastia de reconstrução cirúrgica prepucial como técnica alternativa para tratamento de CCE em cão.

\section{Relato de caso}

Foi atendido um cão da raça PitBull de 7 anos, com pelame branco e pele com pouca pigmentação, com nódulo ulcerado e áreas circulares de eritema em prepúcio, com evolução de três meses, sendo que há 15 dias apresentava sangramento. $\mathrm{O}$ animal possuía comportamento de se expor ao sol e lambeduras das lesões.

Ao exame físico foi observado um nódulo ulcerado em terço proximal lateral direito de prepúcio, de $2,5 \times 3,5 \mathrm{~cm}$, com miíase e nódulos bilaterais (esquerdo $2 \times 3 \mathrm{~cm}$ e direito $0,3 \mathrm{~cm}$ de diâmetro) não ulcerados e eritematosos, espessamento de derme em abdome ventral, na porção hipogástrica (Figura 1). Foi detectado também que o animal possuía pênis infantil e testículos diminuídos para a raça.

Foi realizado hemograma completo e perfil bioquímico sérico (creatinina, proteínas totais, glicose, alaninoamino transferase, fosfatase alcalina). Evidenciou-se trombocitopenia (90.000 plaquetas) e creatinina sérica acima do valor de normalidade (2,9 $\mathrm{mg} / \mathrm{dl})$. Como o paciente possuía histórico de ectoparasitoses, e por suspeitar-se da presença de hemoparasitas aplicou-se uma dose de imizol囚 $(5 \mathrm{mg} / \mathrm{kg}, \mathrm{SC})$, repetida em 15 dias e também utilizou-se a doxiciclina (10mg/kg, PO, BID).

Para se obter o diagnóstico da lesão foi realizada inicialmente a citologia aspirativa com agulha fina (CAAF), apresentando esta resultado inconclusivo. Em seguida, optou-se pela biópsia incisional e histopatologia do fragmento, sendo $o$ nódulo diagnosticado como carcinoma de células escamosas (CCE).

Após o resultado da histopatologia do fragmento obtido pela biópsia incisional, foi realizada a ultrassonografia abdominal para pesquisa de metástase. Descartou-se metástase em linfonodos abdominais e retroperitoneas, mas detectou um aumento renal bilateral com acentuada hiperecogenicidade das corticais, sugestivo de nefropatias e neoplasias, entretanto sem a biópsia renal não foi possível correlacionar ao CCE.

Optou-se então por realizar uma cirurgia plástica conservativa devido à extensão e localização da lesão. Após a exérese do nódulo ulcerado do terço proximal cranial do prepúcio com aproximadamente um centímetro de margem, a pele abdominal cranial e caudal foram divulsionadas, preservando a irrigação principal. A incisão estendeu-se para o abdome lateral por aproximadamente oito centímetros de cada lado, em forma de cunha. Foi mantido o óstio prepucial com aproximadamente dois centímetros de extensão, de forma a conservar a irrigação. Foi orientada a parte caudal do prepúcio ao óstio, evitando desvio peniano durante a sutura de redução de espaço morto. O mesmo procedimento foi realizado em toda extensão abdominal da incisão, durante o avanço cranial e caudal da pele abdominal sobre o prepúcio (Figura 2).

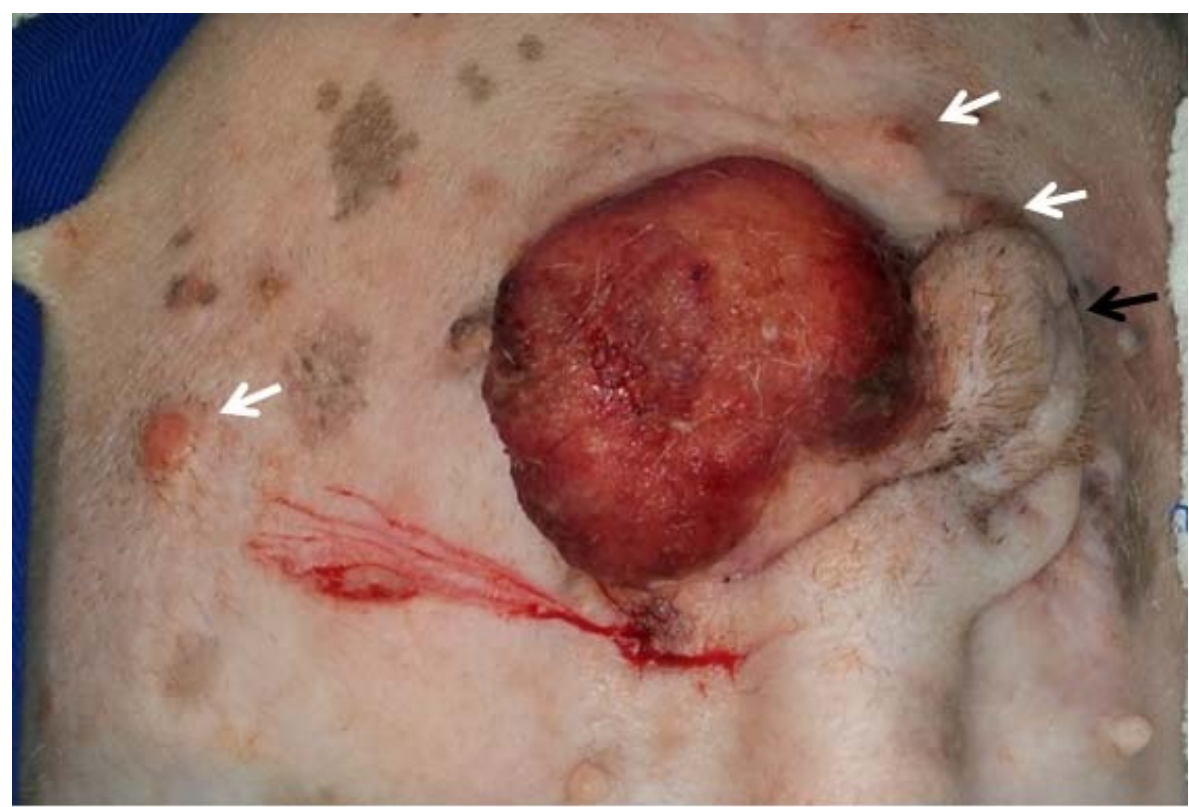

Figura 1: Cão macho da raça Pitbull, com nódulo ulcerado em terço proximal de prepúcio (seta preta) e pequenas nodulações em região abdominal (setas brancas). Fonte: Arquivo pessoal 


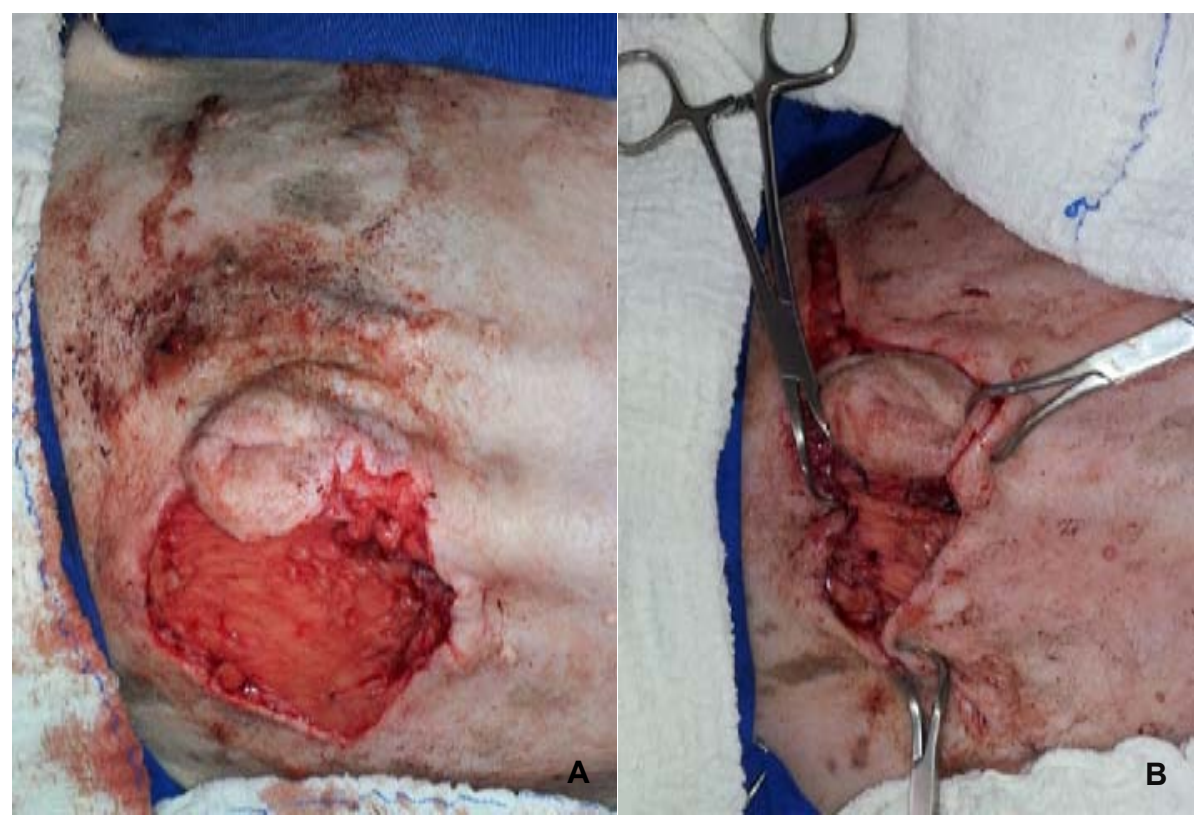

Figura 2: Exérese do nódulo em região proximal de prepúcio $(A)$, remoção dos nódulos laterais em abdome (B), em uma incisão em forma de cunha. Fonte: Arquivo pessoal

Utilizou-se o padrão de sutura walking, para redução de espaço morto, e cushing para aproximação de bordas, ambas com fio poliglactina 910 2-0. Para pele foi utilizado padrão de sutura simples interrompida com náilon 3-0 (Figura 3).

No pós-operatório imediato e por mais dois dias o animal foi mantido hospitalizado com terapia à base de cefalotina $(30 \mathrm{mg} /$ $\mathrm{kg}, \mathrm{IV}, \mathrm{TID})$, tramadol $(3 \mathrm{mg} / \mathrm{kg}, \mathrm{SC}, \mathrm{TID})$, cetoprofeno $(1 \mathrm{mg} / \mathrm{kg}$, SC, SID) e fluidoterapia (1x manutenção) com solução fisiológica ( $\mathrm{NaCl}$ 0,9\%). Após alta hospitalar, foi prescrito cefalexina por 10 dias, tramadol por mais cinco dias e meloxicam $(0,1 \mathrm{mg} / \mathrm{kg}$,
PO, SID) por dois dias. Após 15 dias, o paciente retornou para reavaliação e retirada de pontos.

O exame histopatológico foi repetido, desta vez sendo avaliados os três nódulos excisados e as suas respectivas margens. $O$ resultado foi obtido 30 dias após a cirurgia, onde se confirmou CCE com alto grau de mitose e margens comprometidas. Optouse então por iniciar o tratamento com piroxicam $(0,3 \mathrm{mg} / \mathrm{kg}$, PO, SID) por tempo indeterminado. Após 15 dias o paciente foi reavaliado e dois novos focos neoplásicos foram observados, um em região de axila esquerda e outro em região hipogástrica

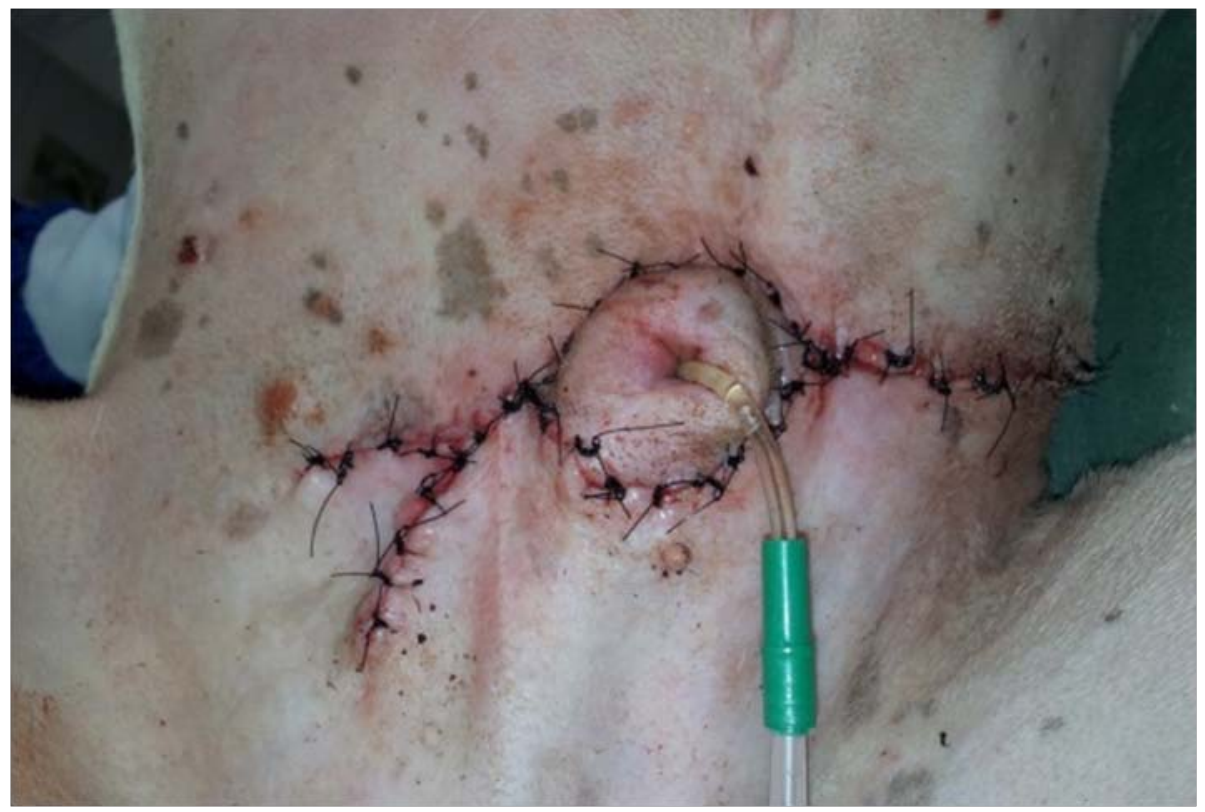

Figura 3: Pós-operatório imediato de exérese de CCE em região proximal de prepúcio de um cão Pitbull. Fonte: Arquivo pessoal. 
ventral de abdome, em lateral esquerda do prepúcio. Decidiuse por realizar novo procedimento cirúrgico, visando a exérese dos nódulos, de forma elíptica e respeitando a margem de três centímetros e a orquiectomia eletiva (Figura 4). Foi utilizado o mesmo padrão de sutura e fios da cirurgia anterior. Prescrito antibioticoterapia e analgesia após a cirurgia por 10 dias; como terapia adjuvante ao tratamento cirúrgico foi mantido o piroxicam por tempo indeterminado.
Neste estudo o cão apresentava nódulos multifocais na primeira avaliação, a CAAF foi inconclusiva, possivelmente pela menor sensibilidade do exame citológico em comparação ao histológico (Vail e Whithrow, 2007). Entretanto, com a confirmação do diagnóstico de CCE, foi indicada a quimioterapia pré-operatória com a finalidade de realizar a citorredução da massa. Essa indicação foi baseada em relatos de tratamento multimodais, onde o paciente apresentou maior tempo livre de doença

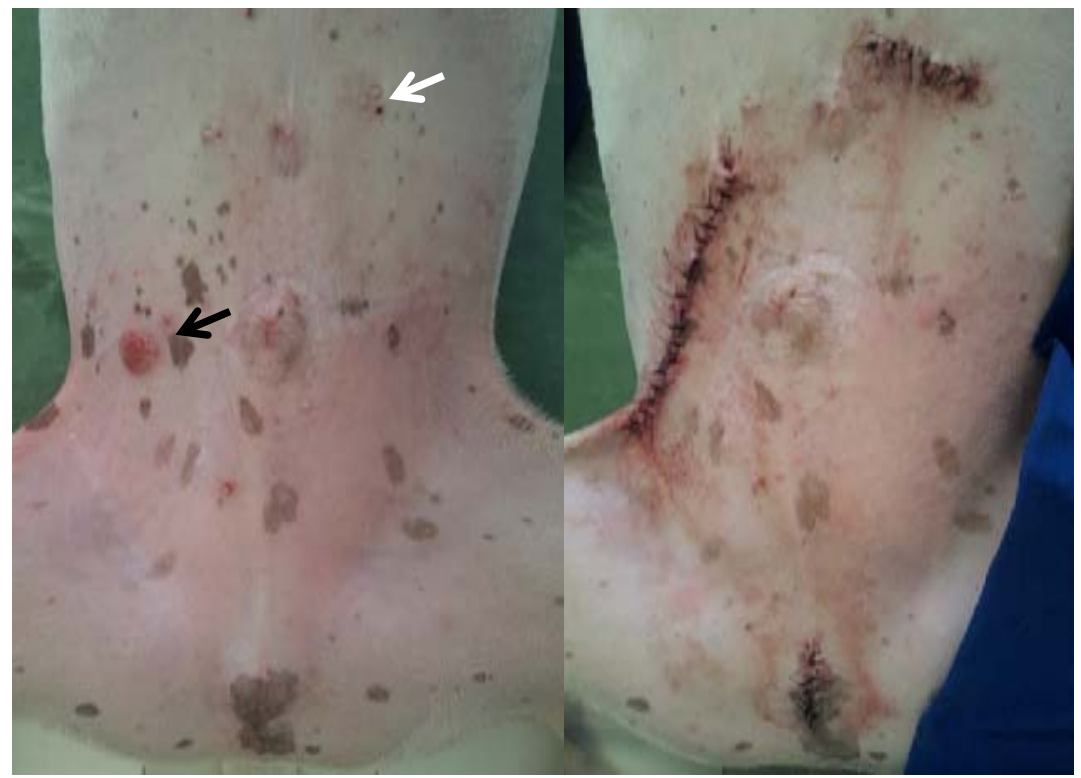

Figura 4: Exérese de nódulos de CCE em abdome lateral - região hipocondríaca esquerda (seta preta) e cranial - região inguinal direita (seta branca) associado à orquiectomia eletiva. Fonte: Arquivo pessoal.

O paciente foi monitorado periodicamente durante um ano após os procedimentos cirúrgicos, sem apresentar sinais de recidivas.

\section{Discussão}

Os fatores predisponentes e desencadeantes das mutações que originam as neoplasias e os melhores tratamentos são alvo de inúmeras pesquisas, tanto na medicina humana, como na veterinária. $O$ tratamento multimodal, com sinergismo de terapias e diminuição de efeitos colaterais, tem sido o objetivo de estudos de alguns pesquisadores (Carvalho et al., 2004; Syrigos et al., 2009). No caso do tratamento de CCE em cães, a quimioterapia tem demonstrado efeito paliativo, como única terapia, ou adjuvante, em terapias multimodais nos estágios avançados com metástase e ulceração, com efeitos de toxidade importantes quando associado à radioterapia (Hume et al., 2009).

A exérese cirúrgica ainda é a primeira opção de tratamento, sendo também considerada em terapias multimodais, em fases posteriores a citorredução (Mcleode Thrall, 1989). A cirurgia em áreas de difícil ressecção é controversa, podendo acarretar em margens comprometidas e complicações no pós-operatório, como hemorragia difusa, deiscência de sutura da uretrostomia e risco de infecções urinárias (Smeak e Newton, 1998; Smeak, 2000; Klein, 2003).
(Langova et al., 2004; Mestrinho et al., 2012). Entretanto, o proprietário autorizou apenas o tratamento cirúrgico devido aos custos. A escolha da técnica cirúrgica conservativa foi em decorrência da possibilidade de complicações pós-cirúrgicas relatadas em penectomias e uretrostomias pré-escrotais (Smeak e Newton 1998; Smeak, 2000). Outro fator levado em consideração foi o ambiente onde o paciente era mantido, que era área rural sem restrição do espaço e ausência de cuidados intensos.

Como o laudo histopatológico revelou áreas contaminadas com alto grau de mitose, optou-se pela quimioterapia neoadjuvante. Dentre as possibilidades decidiu-se pelo piroxicam por apresentar menores efeitos colaterais (Vail e Withrow, 2004) e devido à restrição econômica do proprietário.

O tempo livre de doença após a segunda cirurgia foi de um ano desde a última avaliação, sem novas recidivas. O tratamento conservativo baseado em técnicas de reconstrução plástica associado à quimioterapia adjuvante com piroxicam, não apresentou efeitos colaterais, e comparado a tratamentos multimodais, apresentou uma média de tempo livre de doença maior ou aproximada com a citada na literatura (Mccaw et al., 2000; Langova et al., 2004; Vaile Withrow, 2007; Queiroz et al., 2008; Mestrinho et al., 2012) utilizando procedimento menos cruento, de custo inferior, com menor risco de complicação e preservando a qualidade de vida do paciente. 


\section{Conclusão}

O tratamento cirúrgico baseado em técnicas conservativas e reconstrutivas, associado à quimioterapia adjuvante com

\section{Referências}

ALVES, C.E.F.; VEXENAT, S.C.O.R.; VIDOTTO, V.T.;COSTA, S.S. Carcinoma de células escamosas retrobulbar em um cão. Veterinária E Zootecnia, v.18, n.1, p. 89-92, 2011.

CALAIS, G.; ALFONSI, M.; BARDET, E.;SIRE, C.; GERMAIN, T.; BERGEROT, P.; RHEIN, B.; TORTOCHAUX, J.; OUDINOT, P.; BERTRAND, P. Randomized trial of radiation therapy versus concomitant chemotherapy and radiation therapy for advancedstage oropharynxcarcinoma. Journal of the National Cancer Institute. v. 91, n. 24, p. 2081-2086, 1999.

CARVALHO, A.L.; IKEDA, M.K.;MAGRIN, J.;KOWALSKI, L.P. Trends of oral and oropharyngealcancer survival over five decades in 3267 patients treated in a single institution.Oral Oncology, v. 40, p.71-76, 2004.

COHEN, D. The canine transmissible venereal tumor: a unique result of tumorprogression. Advances in Cancer Research, v. 43, p.75-112, 1985.

DOMINGUEZ-TEJERINA, J.C.; PEÑA, V.; ANEL, L.; CARBAJO, M.; ALEGRE, B. Tumor Venéreotransmisible. ArchivosReproducción Animal, n. 1, p. 42-47, 1996.

FAN, M.T.;LORIMIER,L.P.Tumors of the Male Reproductive System. In: WITHROW, S.J.; VAIL, D.M.; PAGE, R. Withrow and Macewen's Small Animal Clinical Oncology.4 ed. Missouri: W.B. Saunders, 2007, p. 637-648.

FURLANI, J.; DALECK, C.; VICENTI, F.; NARDI, B.A.; PEREIRA, G.T.; SANTANA, A.E.; EURIDES, D.; SILVA, F.A.L.Mastocitoma canino: estudo retrospectivo. Ciência Animal Brasileira, v. 9, n. 1 , p. 242-250,2008.

GONÇALVES, C.M.; MOMESSO, C.G. Resistência ao Sulfato de vincristina em cadela com tumor venéreo transmissível: Revisão literária e relato de caso. In: VI Congresso Paulista de Medicina Veterinária, 2004 São Paulo: SPMV. 2004.

HESS, D.A.; CATCHATOURIAN, R.; ZANDER, R.A.; EPSTEIN, B.R. Intralesional Bacillus Calmette-Guérin Immunotherapy of Canine Veneral Tumors.CancerResearch,v. 37, n. 11, p. 39903994,1977.

HOLMBERG, D. Criocirurgia.In:SLATTER, D.Manual de Cirurgia de Pequenos Animais.3 ed. Barueri:ManoleLtda, 2007, v.1, p. 222-227.

HUME, K.R.; JOHNSON, J.L.;WILLIAMS, L.E. Adverse effects of concurrent carboplatinchemotherapy and radiation therapy in dogs.Journal of Veterinary Internal Medicine,v. 23, n. 1, p.2430, 2009.

JOSEPH, A.; IMPELLIZERI D; GLEN E. Expression of cyclooxygenase-2 in canine nasal carcinomas. The Veterinary Journal, v. 176, n. 3, p. 408-410, 2008.

KLEIN, M.K. Multimodality therapy for head and neck cancer. Veterinary Clinics North America Small Anim Practice, v. 33, n. 3, p. 615-628, 2003. piroxicam, no caso de CCE prepucial, mostrou-se uma opção viável às técnicas mais radicais. As complicações referentes à penectomia e uretrostomia foram evitadas, o que proporcionou melhor qualidade de vida ao animal.

LANGOVA, V.; MUTSAERS, A.J.; PHILLIPS, B.; STRAW, R.Treatment of eight dogs with nasal tumours with alternating doses of doxorubicin and carboplatin in conjunction with oral piroxicam. AustralianVeterinaryJournal, v. 82, n. 11, p. 676-680, 2004.

LAVALLE, G.E; ARAUJO, R.B.; CARNEIRO, R.A.; PEREIRA, L.C. Punção aspirativa por agulha fina para diagnóstico de mastocitoma em cães. Arquivo Brasileirode Medicina Veterinária E Zootecnia, v. 55, n. 4, p. 500-502, 2003.

LAVALLE, G.E.; ARAÚJO, R.B.; CARNEIRO, R.A. Mastocitoma cutâneo canino: revisão de literatura.A Hora Veterinária, v. 23, n. 138, 2004.

MCCAW, D.L.; POPE, E.R.; PAYNE, J.T.; WEST, M.K.; TOMPSON, R.V.; TATE, D. Treatment of canine oral squamous cell carcinomas with photodynamic therapy. British Journal of Cancer,v. 82, n. 7, p. 1297-1299, 2000.

MARTINS, M.;SOUZA, F.; GOBELLO, C.The Canine Transmissible Venereal Tumor: Etiology, Pathology, Diagnosis and Treatment. In: Recent Advances in Small Animal Reproduction, CONCANNON, P.W.; ENGLAND, G.; VERSTEGEN III, J.; LINDE-FORSBERG, C.Recent Advances in Small Animal Reproduction. Ithaca. International Veterinary Information Service (www.ivis.org), 2005. p. A1233.0405.

MCLEOD, D.; THRALL, D.E. The combination of surgery and radiation in the treatment of cancer: a review. Veterinary Surgery,v. 18, n. 1, p. 1-6, 1989.

MESTRINHO, L.A.; BERNARDO, E.; NIZA, M.M.R.E.; LLORETD, A.; BURACCOE, P. Neoadjuvantchemoradiotherapy and surgery as treatment for oral maxillary squamous cell carcinoma in a dog. Australian Veterinary Journal, v. 90, n. 7, p. 264-268, 2012.

NADEAU, M.E.; KITCHELL, B.E.; ROOKS, R.L.; LARUE, S.M. Cobalt radiation with or withoutlow-dose cisplatin for treatment of canine naso-sinus carcinomas. Veterinary Radiology\&Ultrasound, v. 45, n. 4, p. 362-367, 2004.

PARGANA, A.M. Técnicas reconstrutivas em cirurgia oncológica de canídeos e felídeos. 2009. 155 f. Dissertação (Mestrado) Faculdade de Medicina Veterinária - Universidade Técnica de Lisboa, Lisboa, 2009.

PINTO, P.J.; TEIXEIRA, C.B.L.; JÚNIOR, A.R.S. Estudo histopatológico e histoquímico de mastocitomas na região de espírito santo do pinhal. Revista Acadêmica, v. 5, n. 3, p. 265-276, 2007.

QUEIROZ, G.; JULIA, M.; DAGLI, M. Clinical study of cryosurgery efficacy in the treatment of skin and subcutaneous tumors in dogs and cats. VeterinarySurgery, v. 37, n. 5, p. 438-443, 2008.

ROCHA, J.R.; SANTOS, L.M.; TRENTIN, T.C.; ROCHA, F.P.C.; PACHECO, M.D.; FRIOLANI, M. Carcinoma de células escamosas em cães - Relato de caso. Revista Científica Eletrônica de Medicina Veterinária, v. 14, n. 14, 2010.

RODASKY, S.; WERNER, J. Neoplasias de pele. In: DALECK, C.R. Oncologia em Cães e Gatos.São Paulo: Roca Ltda, 1998, p. 253-279. 
ROGERS, K.S. Transmissible venereal tumour.Compendium on Continuing Education for the Practising Veterinarian, v.19, n. 9, p.1036-1045, 1997.

SAKUMA, C.; MATERA, J. M.; VALENTE, N. S. Estudo clínico sobre aplicação do retalho cutâneo pediculado em cirurgia oncológica no cão Clinical study of skin flap application during oncologic surgery in dog. Braz $\mathrm{J}$ vet Res anim Sci, v. 40, $\mathrm{n}$. suplemento 1, 2003.

SMEAK, D.D. Urethrotomy and Urethrostomy in the Dog.Clinical Techniques in Small Animal Practice, v.15, n.1, p. 25-34, 2000.

SMEAK, D.D.; NEWTON, J.D. Canine scrotal urethrostomy. In:BOJRAB, M.J.Current Techniques in Small Animal Surgery.4. ed. Baltimore: Williams \&Wilkins, 1998, p. 465-468.

SOBRAL, R.A.; TINUCCI-COSTA, M.; CAMACHO, A. Ocorrência do tumor venéreo transmissível em cães na região de Jaboticabal. ArsVeterinaria, v.14, n.1, p.1-10,1998.

SOUZA,M.T.; FIGUEIRA, A.R.; IRIGOYEN, F.L.; BARROS, L.S.C. Estudo retrospectivo de 761 tumores cutâneos em cães. Ciência Rural, v. 36, n. 2, p. 555-560, 2006.

SPUGNINI, P.E.; DOTSINSKY, I.; MUDROV, N.; CITRO, G.; D'AVINO, A.; BALDI, A. Biphasic pulses enhance bleomycin efficacy in a spontaneous canine genital tumor model of chemoresistance: Sticker sarcoma. Journal of Experimental \& Clinical Cancer Research,v. 27, n. 58, 2008.
STRAW, R.C.Resection of the nasal planum. In: BOJRAB, M.J. et al. Current techniques insmall animal surgery, 4. ed. Baltimore. Williams \& Wilkins, 1998. p. 343-346.

SYRIGOS, K.N.; KARACHALIOS, D.; KARAPANAGIOTOU, E.M.; NUTTING, M.C.; MANOLOPOULOS, L.; HARRINGTON, J.K. Head and neck cancer in the elderly: an overview on the treatment modalities. Cancer Treatment Reviews,v. 35, p. 237245, 2009.

SZENTIMREY, David. Principles of reconstructive surgery for the tumor patient. Clinical techniques in small animal practice, v. 13, n. 1, p. $70-76,1998$.

THAMM, D.; VAIL, D.Mast Cell Tumor. In: WITHROW, S.J.; VAIL, D.M.; PAGE, R.Withrow and Macewen'sSmall Animal Clinical Oncology. 4. ed. Missouri: W.B. Saunders, 2007, p. 402-424.

VAIL, D.;WITHROW, S.Tumor of the Skin and Subcutaneous Tissues. In: WITHROW, S.J.; VAIL, D.M.; PAGE, R. Withrow and Macewen's Small Animal Clinical Oncology.4 ed. Missouri:W.B. Saunders, 2007, p. 372-401. 